\title{
AVALIAÇÃO DO GANHO DE PESO AJUSTADO PARA 205 DIAS EM BEZERROS DA RAÇA NELORE E MESTIÇOS NELORE x RED ANGUS, SUBMETIDOS AO DESMAME TEMPORÁRIO
}

\author{
Evaluation of weight gain adjusted to 205 days in calves of nellore and \\ crossbred nellore/ red angus, submitted to three temporary weaning
}

\author{
Pedrinho Mariani ${ }^{[a]}$, Weverton Willian Vizentin ${ }^{[a]}$, Leandro Lipinski ${ }^{[b]}$, \\ Marcio Saporski Segui ${ }^{[c]}$, Romildo Romualdo Weiss ${ }^{[\mathrm{d}]}$, Luiz Ernandes Kozicki ${ }^{[c]}$, \\ José Carlos Breda ${ }^{[e]}$, Alisson Bruno Moraes Giacomeli ${ }^{[e]}$
}

\footnotetext{
[a] Zootecnistas da Emater PR, Ponta Grossa, PR - Brasil, e-mail: pmariani@ig.com.br; agrovetvizentin@yahoo.com.br

${ }^{[b]}$ Médico Veterinário do Centro de Ensino Superior dos Campos Gerais (CESCAGE), Ponta Grossa, PR - Brasil, e-mail: leandrolipinski@yahoo.com.br

${ }^{[c]}$ Docentes da Pontifícia Universidade Católica do Paraná (PUCPR), Curitiba, PR - Brasil, e-mail: msegui@uol.com; kozicki.1@pucpr.br

${ }^{[d]}$ Docente da Universidade Federal do Paraná (UFPR),Curitiba, PR - Brasil, e-mail: rrweiss@ufpr.br

[e] Acadêmicos de Medicina Veterinária da Pontifícia Universidade Católica do Paraná (PUCPR), Curitiba, PR - Brasil, e-mail: josecarlosbreda@yahoo.com.br; alissongiacomeli@yahoo.com.br
}

\begin{abstract}
Resumo
No presente experimento objetivou-se avaliar o ganho de peso de bezerros e bezerras da raça nelore e mestiços (nelore x red angus), submetidos a três desmames temporários com duração de 48 horas, realizados a cada 15 dias. A pesquisa foi conduzida em Iguatemi, MS. Para isso, foram constituídos quatro grupos de animais: G1 (26 fêmeas nelore); G2 (28 machos nelore); G3 (20 fêmeas mestiças nelore x red angus fêmea) e G4 (27 machos mestiços nelore x red angus). No experimento, os animais apresentavam pesos iniciais entre 88,8 e 119,3 kg, e permaneciam com a mãe diuturnamente, exceto nos dias em que o tratamento era aplicado. A análise estatística foi executada com os dados de peso ajustados para 205 dias. Concluiu-se que o tratamento empregado não influenciou o ganho de peso dos bezerros ( $p>0,05$ ) nos $G 1, G 2$ e G4, exceto no G3 (fêmeas mestiças nelore $x$ red angus fêmea), em que as mamadas interrompidas influenciaram o ganho de peso em prol do grupo não-tratado $(\mathrm{p}<0,05)$.
\end{abstract}

Palavras-chave: Bovinos de corte. Bezerros nelore e mestiço. Desmame temporário. 


\begin{abstract}
This experiment aimed to evaluate weight gain in calves of nellore and crossbred, submitted to three temporary weaning with duration of 48 hours each, performed in intervals of 15 days. It was assembled four groups of animals: G1 (26 animals female nellore) G2 (28 male nellore) G3 (20 nellore cross/red angus female) and G4 (27 nellore cross/red angus male). In all groups there were control animals and animals subjected to interrupted feeding. In the beginning of the experiment they presented weights ranging from 88.8 to $119.3 \mathrm{~kg}$ and remained with the mother every day, except on the days when treatment was applied. Statistical analysis was done with the data of weight adjusted for 205 days. It was concluded that the animals in group 3 (cross-nellore/red angus female) showed difference $(p<0.05)$ between control and subject to stop breastfeeding, according to which the treated animals had lower weight gain, whereas in the other groups was not statistical difference. It is concluded that the treatment did not influenced the weight gain of calves, except in the group G3.
\end{abstract}

Keywords: Beef cattle. Nellore and crossbred calves. Temporary weaning.

\title{
INTRODUÇÃO
}

A produção de bovinos de corte tem expressivo valor na economia brasileira, em função do significativo volume de consumo interno e das exportações. Os índices financeiros da agropecuária sinalizam que a pecuária brasileira está sendo bastante eficiente, muito embora a realidade tenha demonstrado que tecnicamente existem barreiras, que dificultam a pecuária eficiente e produtiva. Dentre esses entraves ressalta-se o anestro pós-parto longo, que acarreta aumento significativo no intervalo entre partos.

Na pecuária de corte o bezerro é mantido com a mãe, o que lhe permite mamar diuturnamente. A amamentação contínua dos bezerros deprime os centros hipotalâmicos responsáveis pela liberação do hormônio folículo estimulante (FSH) e do hormônio luteinizante (LH), indispensáveis ao desencadeamento do estro, acarretando aumento do período de anestro pós-parto. Este fenômeno ocorre com maior frequência em algumas raças de corte brasileiras, principalmente no bovino de corte tipo zebuíno. Oxenreider e Wagner (1971) e Short et al. (1972) mostraram que a separação do bezerro depois do parto reduz o período de repouso sexual anéstrico. Diversas pesquisas têm demonstrado que a presença do bezerro ao pé da mãe, como ocorre no manejo tradicional (amamentação durante todo o dia), pode exercer efeito negativo sobre o retorno à atividade reprodutiva, prolongando o período de serviço e diminuindo a taxa de eficiência reprodutiva. Segundo Maciel et al. (2001), Borges e Gregory (2003), o desmame temporário exerce significativa influência sobre o desempenho reprodutivo das vacas, quando associado a protocolos hormonais para indução à ciclicidade em vacas de corte no período pós-parto; porém, a aplicação de determinados métodos de manejo, como a colocação de tabuletas para o desmame temporário, podem acarretar doenças nos bezerros, como a síndrome do abscesso pituitário em bezerros, descrita por Loretti et al. (2003) no Rio Grande do Sul.

O desmame precoce é uma técnica utilizada para aumentar os índices reprodutivos do rebanho de cria, com a redução da demanda energética da vaca pela suspensão do aleitamento. A eficiência da transformação de forragem em leite e desse último em crescimento do bezerro é de apenas 5\% a 7\% (ROVIRA, 1996). Entretanto, a vantagem energética e reprodutiva para as fêmeas não pode prejudicar o desenvolvimento dos bezerros. Segundo Restle et al. (1999), quando o consumo de leite é cortado pelo desmame precoce, o bezerro, devido às condições de rúmen, principalmente à baixa capacidade física, não consegue retirar do pasto ingerido a quantidade necessária de nutrientes para manter o mesmo ritmo de ganho de peso que vinha apresentava ao pé da mãe.

Rev. Acad., Ciênc. Agrár. Ambient., Curitiba, v. 7, n. 4, p. 407-413, out./dez. 2009 
Animais jovens após o desmame demonstram ganhos de peso mais eficientes, pois apresentam maior crescimento de tecido muscular em relação ao tecido adiposo. Entretanto, levam mais dias até atingir o maior grau de acabamento de carcaça aceitável (ALMEIDA, et al., 2003). Segundo esses autores, embora o desmame precoce seja eficiente ao incrementar a fertilidade das vacas, o desenvolvimento dos bezerros desmamados precocemente é dependente do manejo alimentar que os mesmos recebem após o desmame.

A raça ou cruzamentos também podem influenciar a produção de leite das vacas. Vários autores têm relatado uma produção de leite e maiores pesos de bezerros ao desmame de vacas cruzadas em relação às vacas de raça pura (CUNDIFF et al., 1974; CARTWRIGHT, 1976; LAMOND, 1976; GREGORY et al., 1992). Ribeiro e Lobato (1988) avaliaram o desempenho de bezerros filhos de vacas primíparas de três grupos raciais: G1 - 3/4 red angus, 1/4 devon; G2 - 3/4 charolês, 1/4 devon; G3 - 1/2 tabapuã, $1 / 2$ devon. O ganho de peso dos bezerros até o desmame e o peso ao desmame foi significativamente superior para os bezerros filhos de vacas do G3, cruzados taurino x zebuíno, em relação aos demais, cruzados taurino x taurino.

A técnica da mamada interrompida é de manejo relativamente fácil e de baixo custo operacional, constituindo-se em alternativa economicamente viável para se melhorar a fertilidade de fêmeas bovinas de corte durante o período pós-parto (EZEQUIEL; KEPLER, 1997). Segundo Almeida, Lobato e Schenkel (2002), o desmame precoce propicia melhor condição corporal às vacas durante o primeiro acasalamento, possibilitando índices reprodutivos significativamente melhores que os do desmame convencional. O acompanhamento do desempenho de bezerros nos seus primeiros meses de vida tem fundamental importância uma vez que esse exerce influência sobre a posterior produção dos animais. Restle et al. (2005) verificaram bezerras com taxas de ganho de peso menores que $350 \mathrm{~g} /$ dia até os sete meses de idade, enquanto as vacas produziam maiores quantidades de leite (4,83 vs 3,71 L/dia), total de gordura (47,4 vs 35,9 kg), lactose (52,6 vs 39,7 kg), extrato seco total (140,4 vs 107,2 kg) e extrato seco desengordurado (94,8 vs $71,2 \mathrm{~kg}$ ), além de bezerros com maiores ganhos de peso (717 vs $617 \mathrm{~g} / \mathrm{dia}$ ) e mais pesados ao desmame (189,0 vs 166,1 kg) que as vacas que, quando bezerras, apresentaram taxas de ganho de peso acima de $350 \mathrm{~g} /$ dia.

A presente pesquisa teve por finalidade avaliar o ganho de peso de bezerros, submetidos a três desmamas temporárias (mamadas interrompidas), em rebanhos nelore e mestiços nelore, ao fim de 205 dias, quando ocorreu o desmame definitivo.

\section{MATERIAL E MÉTODOS}

O experimento foi desenvolvido em fazenda de bovino de corte, situada em Iguatemi (MS). Foram utilizados 101 bezerros com 100 dias de idade em média, alocados ao acaso em grupos experimentais, controles e grupos com mamadas interrompidas, conforme sexo e raça, da seguinte forma: G1 - fêmeas puras da raça nelore, G2 - machos puros da raça nelore, G3 - fêmeas mestiças nelore x red angus e G4 - machos mestiços nelore x red angus, como demonstrado na Tabela 1. Os animais, no inicio do experimento, pesavam entre 88,8 e $119,0 \mathrm{~kg}$ e eram separados de suas respectivas mães no período da manhã, para aplicação do teste. Os animais separados permaneciam em um piquete especifico, à distância aproximada de $15 \mathrm{~m}$ das respectivas mães, visualizando-se mutuamente, mas sem contato durante o período de 48 horas. Os bezerros eram mantidos a pasto constituído predominantemente por Brachiaria brizanta, além de sal energético e de água ad libitum. Após 48 horas de separação, os grupos de bezerros retornavam às mães, através de um corredor. Esse protocolo de manejo foi repetido a cada 15 dias por três vezes consecutivas. Os animais testados, apesar de constituírem grupos homogêneos (todos os bezerros em lactação) não eram da mesma idade. Para analisar os dados, os pesos foram ajustados para 205 dias, objetivando uniformizar a idade e eliminar defeitos de amostragem.

Rev. Acad., Ciênc. Agrár. Ambient., Curitiba, v. 7, n. 4, p. 407-413, out./dez. 2009 
Foi aplicada a seguinte fórmula de cálculo para ajustar o peso para 205 dias:

$$
P A D=\frac{P D-P N}{N D} X 205+P N
$$

Em que:

PAD = Peso ajustado ao desmame

$\mathrm{PD}=$ Peso ao desmame

$\mathrm{PN}=$ Peso ao nascer

ND = Número de dias

TABELA 1 - Número e peso médio inicial de bezerros da raça nelore e mestiços nelore / red angus, submetidos ao desmame interrompido por 48 horas quinzenalmente $(\mathrm{n}=101)$

\begin{tabular}{lcc}
\hline Grupos & Controle & Mamada interrompida \\
\hline Nelore fêmea (n) & 10 & 16 \\
Peso (kg) & 95,2 & 88,8 \\
Nelore macho (n) & 11 & 17 \\
Peso (kg) & 94,2 & 98,0 \\
Fêmeas mestiças nelore x red (n) & 8 & 12 \\
Peso (kg) & 117,0 & 115,5 \\
Machos mestiços nelore x red (n) & 9 & 18 \\
Peso (kg) & 119,3 & 119,2 \\
\hline
\end{tabular}

Executou-se uma pesagem inicial dos bezerros e outra após os três apartes (retiradas dos bezerros das mães). Os dados obtidos foram tratados estatisticamente, empregando-se o Teste $t$ de Student $(\alpha=0,05)$, visando a verificar as diferenças de ganho de peso dos animais controles e dos submetidos ao desmame interrompido. Os dados de peso foram ajustados para 205 dias.

\section{RESULTADOS E DISCUSSÃO}

Os resultados do presente trabalho encontram-se nas Tabelas 2 e 3.

TABELA 2 - Ganho de peso médio diário (kg) de bezerros da raça nelore e mestiços nelore/red angus, dos 90 dias até o desmame, ajustado para 205 dias

\begin{tabular}{|c|c|c|c|c|c|c|c|c|}
\hline \multirow[b]{4}{*}{$\mathrm{n}$} & \multicolumn{4}{|c|}{ Nelore } & \multicolumn{4}{|c|}{ Mestiços Nelore x Red angus } \\
\hline & \multicolumn{2}{|c|}{ Fêmea } & \multicolumn{2}{|c|}{ Macho } & \multicolumn{2}{|c|}{ Fêmea } & \multicolumn{2}{|c|}{ Macho } \\
\hline & Controle & MI & Controle & MI & Controle & MI & Controle & MI \\
\hline & 10 & 16 & 11 & 17 & 8 & 12 & 9 & 18 \\
\hline $\begin{array}{l}\text { Ganho de } \\
\text { peso diário } \\
(\mathrm{x} \pm \mathrm{s})\end{array}$ & $\begin{array}{r}0,66 \\
\pm 0,07\end{array}$ & $\begin{array}{r}0,62 \\
\pm 0,08\end{array}$ & $\begin{array}{r}0,73 \\
\pm 0,11\end{array}$ & $\begin{array}{r}0,66 \\
\pm 0,10\end{array}$ & $\begin{array}{r}0,81 \\
\pm 0,10\end{array}$ & $\begin{array}{r}0,73 \\
\pm 0,07\end{array}$ & $\begin{array}{r}0,89 \\
+0,12\end{array}$ & $\begin{array}{r}0,78 \\
\pm 0,14\end{array}$ \\
\hline
\end{tabular}

MI = mamada interrompida.

Rev. Acad., Ciênc. Agrár. Ambient., Curitiba, v. 7, n. 4, p. 407-413, out./dez. 2009 
Avaliação do ganho de peso ajustado para 205 dias em bezerros da raça nelore e mestiços nelore $\mathrm{x}$ red angus, submetidos ao desmame temporário

TABELA 3 - Peso (kg) ajustado para 205 dias de fêmeas nelore, machos nelore, fêmeas mestiças nelore x red angus e machos mestiços nelore $\mathrm{x}$ red angus, grupos controles e submetidos às mamadas interrompidas por 48 horas seguidas, por três vezes consecutivas, quinzenalmente

\begin{tabular}{llc}
\hline Grupos & $\begin{array}{c}\text { Média } \\
\text { Controle }\end{array}$ & $\begin{array}{c}\text { Média } \\
\text { Desmama interrompida }\end{array}$ \\
\hline (G1) Fêmeas nelore & $169,24 \mathrm{a}$ & $161,27 \mathrm{a}$ \\
(G2) Machos nelore & $174,93 \mathrm{~b}$ & $167,85 \mathrm{~b}$ \\
(G3) Fêmeas mestiças nelore x red angus & $196,6 \mathrm{c}$ & $183,34 \mathrm{~d}$ \\
(G4) Machos mestiços nelore x red angus & $202,86 \mathrm{e}$ & $188,97 \mathrm{e}$ \\
\hline
\end{tabular}

Letras diferentes na mesma linha indicam significância.

Ao se observar o dado da tabela 2, verifica-se que não houve diferença de peso médio diário entre os grupos de bezerros. Williams (1991) e Fanning et al. (1995), trabalhando com bezerros das raças angus ou hereford, relatam diferenças significativas de peso entre bezerros submetidos a um desmame interrompido e bezerros não submetidos ao tratamento, nas 48 horas que se seguiram ao tratamento. Porém, eles não observaram diferenças significativas de peso entre os grupos, à época do desmame ( $p>0,10)$, confirmando relatos de Beck et al. (1979), Wettermann et al. (1986) e de Makarechian e Arthur (1990). Não foi objetivo da presente pesquisa verificar os pesos de cada grupo constituído após cada shang executado, mas sim a diferença de peso após os três desmames interrompidos. Ao se comparar os grupos animais, ajustando-se o peso para os 205 dias, não se constatou diferença entre os grupos G1, G2 e G4; porém, verificou-se diferença significativa entre os animais tratados e os controles do G3 (fêmeas mestiças nelore com red angus), ocorrendo significativa perda de peso no grupo desmame interrompido (Tabela 3). São escassos os relatos sobre a execução de desmame interrompido com referência ao peso aos 205 dias, como o executado nesta pesquisa. Como observado, é provável que a discrepância entre os dados do presente experimento, verificada no G3, e os relatos de Fanning et al. (1995) ao se consumar o desmame dos animais se deva ao fato de que nesta pesquisa houve três manejos de desmame interrompido a cada 15 dias, e não a um somente, como no experimento feito pelos autores supracitados. Neste experimento, os animais tratados do G3 mostraram-se mais sensíveis ao shang que nos outros grupos.

Com relação ao retorno da atividade ovariana cíclica das vacas lactantes, submetidas ao aparte dos filhos, os resultados demonstraram-se muito promissores em prol dos grupos de desmama interrompida; porém, esse não foi o objetivo do presente experimento.

Os dados obtidos na presente pesquisa permitiram concluir que a prática de três desmames interrompidos nos grupos bezerras nelore fêmea, bezerros nelore e mestiços nelore $\mathrm{x}$ red angus não influenciou o ganho de peso em relação aos respectivos controles, exceto no grupo de bezerras nelore $\mathrm{x}$ red angus, no qual houve diferença $(\mathrm{p}<0,05)$ em prol do grupo controle.

\section{REFERÊNCIAS}

ALMEIDA, L. S. et al. Idade de desmame e suplementação no desenvolvimento e em características de carcaças de novilhos de corte. Revista Brasileira de Zootecnia, Viçosa, v. 32, n. 6, p. 17131721, 2003.

ALMEIDA, L. S. et al. Data de desmame e desempenho reprodutivo de vacas de corte. Revista Brasileira de Zootecnia, Viçosa, v. 31, n. 3, p. 1223-1229, 2002.

BECK, T. W. et al. Influence of 48 hour calf separation on milk production and calf growth in range cows. Theriogenology, Amsterdam, v. 11, n. 5, p. 367-373, 1979. 
BORGES, J. B. S.; GREGORY, R. M. Indução à atividade cíclica ovariana pós-parto em vacas de corte submetidas à interrupção temporária do aleitamento associada ou não ao tratamento com norgestomet-estradiol. Ciência Rural, Santa Maria, v. 33, n. 6, p. 1105-1110, 2003.

CARTWRIGHT, T. C. Comparación entre vacas F1 con las de raza pura y otras cruzas. In: KOGER, M.; CUNHA, T. J.; WARNICK, A. C. (Ed.). Cruzamientos en ganado vacuno de carne. Montevideo: Hemisferio Sur, 1976. p. 62-80.

CUNDIFF, L. V. et al. Effects of heterosis on maternal performance and milk production in Hereford, Angus and Shorthorn cattle. Journal of Animal Science, Stanford, v. 38, n. 4, p. 728 745, 1974.

EZEQUIEL, L. R.; KEPLER, E. F. Efeito da separação temporária do bezerro após a remoção do implante de norgestomet na manifestação de cio. In: REUNIÃO ANUAL DA SOCIEDADE BRASILEIRA DE ZOOTECNIA, 34., 1997, Juiz de Fora. Anais... Juiz de Fora: SBZ, 1997. p. 401-405.

FANNING, M. D. et al. Reproductive performance of synchronized beef cows as affected by inhibition of suckling with nose tags or temporary calf removal. Theriogenology, Amsterdam, v. 44 , n. 5 , p. $715-723,1995$.

GREGORY, K. E. et al. Breed effects and heterosis in advanced generations of composite populations for reproduction and maternal traits of beef cattle. Journal of Animal Science, Stanford, v. 70, n. 3, p. 656-672, 1992.

LAMOND, D. R. Indices de reproducción y destete de ganado de raza pura y cruza en Australia y Nueva Zelandia. In: KOGER, M.; CUNHA, T. J.; WARNICK, A. C. (Ed.). Cruzamientos en ganado vacuno de carne. Montevideo: Hemisferio Sur, 1976. p. 190-201.

LORETTI, A. P. et al. Síndrome do abscesso pituitário em bezerros associada ao uso de tabuleta nasal para desmame interrompido. Pesquisa Veterinária Brasileira, Brasília, v. 23, n. 1, p. 39-46, 2003.

MACIEL, M. N. et al. Programa hormonal associado ao desmame temporário, na indução de ovulação em vacas de corte durante o pós-parto. Ciência Rural, Santa Maria, v. 31, n. 3, p. 473478, 2001.

MAKARECHIAN, M.; ARTHUR, P. F. Effects of body condition and temporary calf removal on reproductive performance of range cows. Theriogenology, Amsterdam, v. 34, n. 3, p. 435-443, 1990.

OXENREIDER, S. L.; WAGNER, W. C. Effect of lactation and energy intake on postpartum ovarian activity in the cow. Journal of Animal Science, Stanford, v. 33, n. 5, p. 1026-1031, 1971.

RESTLE, J. et al. Desenvolvimento de bovinos de corte de diferentes grupos genéticos desmamados aos 3 ou 7 meses de idade. Revista Brasileira de Zootecnia, Viçosa, v. 28, n. 5, p. 1023-1030, 1999.

RESTLE, J. et al. Efeitos da taxa de ganho de peso pré-desmama de bezerras de corte e do nível nutricional pós-parto, quando vacas, sobre a produção e composição do leite e o desempenho de bezerros. Revista Brasileira de Zootecnia, Viçosa, v. 34, n. 1, p. 197-208, 2005. 
RIBEIRO, A. M. L.; LOBATO, J. F. P. Produtividade e eficiência reprodutiva de três grupos raciais de novilhas de corte. II. Desenvolvimento da progênie até o desmame. Revista Brasileira de Zootecnia, Viçosa, v. 17, n. 6, p. 509-515, 1988.

ROVIRA, J. M. Manejo nutritivo de los rodeos de cría en pastoreo. Montevideo: Hemisferio Sur, 1996.

SHORT, R. E. et al. Effects of suckling and mastectomy on bovine postpartum reproduction. Journal of Animal Science, Stanford, v. 34, n. 1, p. 70-74, 1972.

WETTEMANN, R. P. et al. Reproductive performance of postpartum beef cows after short-term calf separation and dietary energy and protein supplementation. Theriogenology, Amsterdam, v. 26, n. 4, p. 433-443, 1986.

WILLIAMS, G. L. Substituting alien cohabitation for complete temporary weaning in estrus synchronization schemes using Syncro-Mate-B. Texas Agricultural Research and Extension Information Report, Beeville, v. 91, n. 1, p. 62-64, 1991.

Recebido: 01/07/2009

Received: 07/01/2009

Aprovado: 16/08/2009

Approved: 08/16/2009

Rev. Acad., Ciênc. Agrár. Ambient., Curitiba, v. 7, n. 4, p. 407-413, out./dez. 2009 